\title{
tic\&société
}

Vol. 10, N 2-3 | 2ème semestre 2016 - 1er semestre 2017

Spécial varia

\section{Radio musicale, prolongements numériques et régimes d'interactivité. Le cas de Nostalgie}

Séverine EQUOY HUTIN

\section{(2) OpenEdition \\ Journals}

Édition électronique

URL : http://journals.openedition.org/ticetsociete/2125

DOI : 10.4000/ticetsociete. 2125

Éditeur

Association ARTIC

Référence électronique

Séverine EQUOY HUTIN, «Radio musicale, prolongements numériques et régimes d'interactivité. Le cas de Nostalgie », tic\&société [En ligne], Vol. 10, № 2-3 | 2ème semestre 2016 - 1er semestre 2017, mis en ligne le 30 avril 2017, consulté le 19 avril 2019. URL : http://journals.openedition.org/ ticetsociete/2125; DOI : 10.4000/ticetsociete.2125 
tic\&société - 10(2-3), 2016-2017

\section{Radio musicale, prolongements numériques et régimes d'interactivité. Le cas de Nostalgie}

\section{Séverine Equoy Hutin}

Séverine Equoy Hutin est maître de conférences en Sciences de l'Information et de la Communication à l'université de Bourgogne Franche-Comté. Elle est membre du pôle Conception Création Médiation du laboratoire ELLIADD (Édition, Littératures, Langages, Informatique, Arts, Didactique, Discours) et coresponsable de l'axe "Communication, Frontières médiatiques et pratiques sociales ». Rédactrice en chef de la revue Semen et membre du GRER, elle mène ses recherches dans une perspective sémiopragmatique en Sciences de l'Information et de la Communication. Ses travaux portent notamment sur les mutations de l'écriture radiophonique en général et en particulier à l'ère du numérique. Celles-ci sont appréhendées sous l'angle des transferts médiatiques, de la circulation-altération des discours et de la mise en scène des savoirs et de la culture populaire par des dispositifs complémentaires. severine.equoy-hutin@univ-fcomte.fr 
Radio musicale, prolongements numériques et régimes d'interactivité. Le cas de Nostalgie

\section{Radio musicale, prolongements numériques et régimes d'interactivité. Le cas de Nostalgie}

Résumé : Les prolongements des radios sur le web proposent de sortir de l'exclusivité du sonore et de la logique de flux au profit d'une logique de stock et d'un accès direct, délinéarisé, à l'information. L'interactivité constitue la principale promesse du web 2.0: d'un régime d'écoute, on passe à un ensemble de propositions audiovisuelles qui décontextualisent les contenus et procèdent par fragmentation, mise à disposition, agrégation. Cette remédiation implique de nouvelles formes d'écritures, de nouvelles modalités de perception, de consommation et une nouvelle relation au média. Si l'interactivité n'est pas étrangère à la radio, le cas des radios musicales est particulier. Les grilles de programmation contiennent peu de " talk » et peu d'espace d'interaction. De plus, les radios musicales subissent la concurrence de " nouveaux médiateurs " de la musique. Dans ce contexte, on s'intéresse à la radio Nostalgie, avec l'hypothèse que ses prolongements numériques (application mobile et site internet) mettent en œuvre de nouveaux moyens d'entrer en contact avec la musique.

Mots-clés : radio musicale, prolongements numériques, formes d'écriture, interactivité.

Abstract: Web radio asks the listener to set aside the exclusivity of sound and a logic of flow in favor of a logic of storage and direct, non-linear access to information. Interactivity is the main promise of Web 2.0: listening to an audiovisual offer is replaced by a system that decontextualizes content and favours fragmentation, availability and aggregation. This re-mediation involves new forms of writing, new modalities of perception and consumption, as well as a new relation with the media. Radio has lengthy experience with interactivity but the case of musical radio is particular in that its programming grid doesn't offer much space for "talk" or interaction. Furthermore, musical radio must compete with "new mediators" of music. In this context, we focus on the case of the French radio station Nostalgie, with the hypothesis that its various digital extensions (mobile application and web site) implement new ways to discover and/or enjoy music.

Keywords: musical radio, digital extensions, forms of writing, interactivity. 
Resumen: Las prolongaciones de las radios en la web suponen el paso de la exclusividad sonora y de la lógica del flujo, en beneficio de una lógica de almacenamiento y de un acceso directo, no lineal, a la información. La interactividad constituye la principal promesa de la Web 2.0 : se pasa de un régimen de escucha a un conjunto de ofertas audiovisuales que sacan de su contexto los contenidos y proceden por fragmentación a la disposición y a la agregación. Esta remediación implica nuevas formas de escritura, nuevas modalidades de percepción, nuevos consumos, así como una nueva relación con el medio de comunicación. La interactividad no es extraña a la radio, pero en el caso de las radios musicales dicha interactividad es especial. Las parrillas de programación contienen poco 'talk' y poco espacio para la interacción. Además, las radios musicales sufren la competencia de 'nuevos mediadores' de la música. En este contexto, este texto se interesa por la radio Nostalgie, con la hipótesis de que sus prolongaciones digitales (aplicación móvil y página internet) inauguran nuevas formas de entrar en contacto con la música.

Palabras clave : radio musical, diversificación digital, formas de escritura, interactividad. 
Radio musicale, prolongements numériques et régimes d'interactivité. Le cas de Nostalgie

La radio a connu son tournant numérique: les prolongements sur le web proposés par les stations (sites, pages Facebook, applications mobiles...) proposent de sortir de l'exclusivité du sonore et de la logique de flux pour adopter une " logique de stock qui favorise un accès direct, délinéarisé, à l'information » (Saint Martin et Crozat, 2007). Ce passage au numérique implique l'édition de contenus qui « augmentent » le flux sonore. L'interactivité constitue la principale promesse du web 2.0 et des technologies de l'information et de la communication. Elle conditionne l'accès à ces contenus devenus disponibles: dans le cas de la communication radiophonique, l'enjeu réside dans le passage d'un régime d'écoute à un régime d'audiovision interactif, c'est-à-dire à un ensemble de propositions audiovisuelles qui décontextualisent et délinéarisent les contenus de la programmation initiale en procédant par fragmentation, mise à disposition, agrégation.

Par opposition aux radios dites "de programmes", les radios musicales sont le plus souvent cantonnées à la diffusion d'un flux musical quasi ininterrompu. Elles privilégient soit des styles de musique (Radio Classique ${ }^{1}$ ) soit un périmètre linguistique (la chanson francophone sur $M F M^{2}$ ) soit des périodes balisées (les années 1970-1980-1990 sur Nostalgie ${ }^{3}$ ). La participation de l'auditeur y reste très limitée et localisée. Considérant ces deux paramètres, on peut formuler l'hypothèse que les prolongements numériques d'une radio musicale et les régimes d'interactivité portée par les organisations sémiotiques issues du processus de remédiation proposent de nouvelles formes d'écriture radiophonique et complémentent ses fonctions initiales. En d'autres termes, on postule que les formes d'écritures qui émergent des dispositifs informatisés mettent en scène et exacerbent le principe d'interactivité au sein duquel se reconfigure le sonore. Afin d'observer les modalités de cette mise en visibilité dans une perspective sémiopragmatique, deux lieux numériques de la station de radio Nostalgie seront examinés : l'application mobile et le site internet.

\footnotetext{
${ }^{1}$ Radio Classique est une radio privée française thématique à vocation nationale : elle diffuse principalement de la musique classique principalement de la musique classique. Sa grille contient aussi des tranches d'informations, consacrées à l'actualité économique et politique.

${ }^{2}$ MFM est une radio privée française thématique spécialisée dans la diffusion de chansons françaises. Elle dispose de 100 fréquences en France.

${ }^{3}$ II s'agit ici de la station Nostalgie, diffusée sur le réseau hertzien français et en modulation de fréquence (FM).
} 


\section{Intermédialité, nouvelles écritures et interactivité}

\subsection{Remédiation et écritures émergentes}

L'écriture radiophonique met en scène différents éléments " organisés, construits, élaborés selon certaines lignes d'orientation par la volonté d'un producteur ou d'un auteur radiophonique " (Richard, 1985, p. 30). Elle recrée "l'illusion d'une continuité cohérente, d'un temps plus intense: le temps de l'écoute » (Saint Martin et Crozat, 2007, p. 7). Sa fonction première est de baliser l'écoute et de la structurer afin de capter l'attention de l'auditeur. Ce balisage passe par une scénographie d'éléments disparates dans une forme de continuité qui suscite l'image et l'imaginaire. Ainsi, l'efficacité de l'écriture radiophonique provient de la capacité de l'acteur professionnel à « ruser avec tous les moyens d'ex-pression techniquement possibles pour créer l'illusion de corps, d'objets, d'apparences, et pour persuader de leur réalité, de leur vie » (Richard, 1985, p. 7).

L'intermédialité et ses dynamiques de passage constituent l'essence de la vie des médias qui naissent, s'affirment et se redéfinissent dans leurs interrelations. La remédiation (Bolter et Grusin, 2000) désigne en ce sens les phénomènes de remodelage des formes médiatiques antérieures et engendre des formes et des organisations sémiotiques nouvelles. Dans ce contexte, des modalités inédites de perception, d'intellection et de consommation du média émergent. Cette rencontre génère des phénomènes irréductibles à la simple addition des propriétés singulières des deux médias concernés; en effet, le processus combinatoire engendre du nouveau qui « émerge à l'intérieur de l'existant, de pratiques anciennes profondément enracinées à partir desquelles de l'inattendu peut se produire » (Angé et Renaud, 2012, p. 36-37). Le prolongement requiert indéniablement une source et interroge par là même la relation entre l'ancien et le nouveau. En passant de l'autonomie à une situation d'inclusion et de reconfiguration, la radio rencontre un nouveau dispositif éditorial et participe d'un processus de rééditorialisation qui prolonge la vie hertzienne du son en mettant en œuvre un certain « mode d'intervention » (Jeanneret et Souchier, 2005, p. 4). Remédiée sur le web, la radio s'inscrit dans différents lieux (sites de station, blogues, chaînes YouTube, applications mobiles, pages Facebook...) et se confronte à des possibilités nouvelles et diverses (écoute en direct, radio filmée, podcasts, photos, liens vers des blogues ou vers les réseaux sociaux...). Si la radio «trouve dans le direct son atout le plus solide, sa rareté la plus précieuse " (Glevarec, 2014 , p. 23), on peut se questionner sur le devenir des contenus décontextualisés de la programmation: quel statut 
Radio musicale, prolongements numériques et régimes d'interactivité. Le cas de Nostalgie

acquièrent-ils dans cette configuration nouvelle? Quelles modalités d'éditorialisation et d'écriture les différents prolongements proposent-ils ?

\section{2. Écriture radiophonique et interactivité}

Le numérique constitue un facteur et un levier d'efficacité pour ces nouvelles formes d'écriture dont nous postulons l'existence. La remédiation de l'hertzien au numérique s'effectue dans le cadre d'une promesse d'interactivité qui fait partie du contrat de communication et suppose à la fois réciprocité, flexibilité, contrôle, personnalisation et égalité. Cette promesse va de pair avec le développement des machines interactives «qui peuvent dialoguer avec l'environnement et dont le programme peut évoluer en temps réel en fonction des modifications apportées par les usagers " (Lejealle, 2010, p. 7).

Dans ce cadre, le rôle de l'usager peut aller de la simple consommation jusqu'à la création de contenus. La promesse d'interactivité se concrétise le plus souvent sur le mode d'une interactivité fermée (Manovich, 2010). Si, selon Manovich, l'interactivité ouverte permet à l'usager de modifier des éléments, voire l'ensemble de la structure, l'interactivité fermée renvoie, quant à elle, à des parcours préprogrammés que l'usager peut décider d'emprunter ou non afin d'accéder à différents contenus. Dans ce cas, il n'a pas prise sur la structure considérée stable. Manovich considère que l'« interactivité fermée » est le propre de la plupart des produits des nouveaux médias. L'usager suit des parcours et navigue en composant, à partir des assemblages proposés, sa version du texte. II trouve une flexibilité en termes d'apport d'informations, de manipulation et de parcours possibles (Saint Martin et Crozat, 2007 ; Crozat et al., 2011) dans un échange avec la machine qui s'effectue sur la base de ces parcours et de ces narrations déjà construites (Souchier, 2012).

L'interactivité entendue comme «intelligence d'accès reposant sur une structure conceptuelle de l'ensemble des contenus dont l'hypertexte, le multicritères ou l'arborescence sont emblématiques " (Paquienséguy, 2011, p. 3) peut être appréhendée sous l'angle des compétences de lecture et d'écriture. L'interactivité constitue en ce sens un des composants de l'écriture, et le multimédia s'en distingue alors en tant que "mode d'agencement des vecteurs de transmission de ces contenus qui caractérise leurs concrétisations sensibles (images, son, animation, 3D...) » (ibid.). Ainsi, dans le passage au numérique, l'interactivité devient un "possible " pour l'écriture radiophonique et les modalités éditoriales mises en œuvre: la rééditorialisation passe donc certes par la mise à disposition 
(recontextualisation), mais aussi par une «mobilisation des modalités d'interaction ouvertes par le numérique " (Crozat et al., 2011, p. 56).

Le principe d'interactivité n'est pas totalement étranger au média radiophonique : les premières propositions d'interaction avec l'auditeur remontent en France à 1927 (Deleu, 2006) et le don de la parole aux auditeurs est devenu une pratique familière à la radio : les émissions de libre antenne lancées par Skyrock en 1991 deviennent même une priorité pour les stations généralistes dans les années 1990. Toutefois, les manifestations à l'antenne des auditeurs et les possibilités d'action sur la programmation restent limitées: le plus souvent filtrées par une équipe de standardistes, elles sont surtout localisées dans les émissions de libre antenne, les magazines de service (Schmitt, 2009) et les jeux. Dans le cas des radios musicales, la situation est particulière, dans la mesure où ces espaces sont encore plus limités.

\subsection{Dispositif interactif dans les écrits d'écran}

Selon Jeanneret et Souchier (2005), «la pratique des médias informatisés a ceci de particulier en effet qu'elle s'effectue à partir de la lecture et de l'écriture » (p. 5). L'analyse des médias informatisés a permis de mettre en avant l'imbrication entre techniques (opérativité) et langage (symbolique), d'une part, et les processus de « textualisation des pratiques sociales " inhérentes aux dispositifs techniques d'autre part. La sémiotique des écrits d'écran qui a forgé les concepts d'architextes, de signes passeurs et de textiels (Jeanneret et Tardy, 2007) a montré que « la dimension sociale de l'usage est en effet modélisée dans l'écriture même des logiciels » (ibid., p. 26). Des hypothèses d'usages sont ainsi paramétrées et suggèrent, voire anticipent, le social qui, à son tour, rétroagit sur le technique. De ce fait, l'architexte est un modèle formulaire pour la production de textes qui préconfigure l'édition des contenus (Bonaccorsi, 2013): il constitue un niveau du dispositif, fournit un gabarit et le soumet à la production puis à la pratique. L'architexte porte des traces d'initiative et mobilise " une conception de ce qu'un usager peut faire avec telle ou telle forme sémiotique » (Jeanneret et Tardy, 2007, p. 27). Or les usagers peuvent ne pas s'emparer nécessairement des prescriptions qui leur sont faites: s'ils se trouvent face à des propositions d'interactivité (recherche, consultation, manipulation, partage, commentariat, etc.), il n'en reste pas moins que la machine répond à une stimulation humaine (Lejealle, 2010).

Si un dispositif interactif intègre nécessairement des représentations de l'usager et des normes d'usage, il en va de 
Radio musicale, prolongements numériques et régimes d'interactivité. Le cas de Nostalgie

même pour l'objet de valeur (Patrin-Leclère et al., 2007) qu'il met en scène (par exemple la musique pour une radio musicale). Des travaux consacrés par exemple à l'écriture du journal radiophonique et à l'incursion d'un logiciel de gestion du journalisme d'actualité dans le passage au numérique de la station France Inter ont montré que « les spécificités du média radio deviennent ainsi invisibles dans un architexte qui affirme le primat du journalisme d'actualité " (Patrin-Leclère et al., 2007, p. 67). En d'autres termes, l'architexte cristallise une autre forme d'écriture de l'actualité qui s'intègre au processus de production de l'information en le réorganisant et en établissant une certaine relation avec le média d'origine. Dans le cas qui nous concerne, il ne s'agit pas d'une écriture d'information journalistique, mais de la diffusion de contenus musicaux dans un processus qui complète la programmation musicale hertzienne et épaissit le flux sonore. Si, par exemple, les sites ou les pages d'émissions consacrées à l'histoire sur les réseaux sociaux (Chauvin Vileno et Equoy Hutin, 2016) alimentent l'épaisseur documentaire du texte source dans un processus de mise en visibilité en lien avec la stratégie de la station et du groupe auquel elle appartient, qu'en est-il des contenus diffusés sur les radios musicales? Dans quelle mesure la rencontre entre la médiation informatique et l'objet de valeur " musique " exprime-t-elle un ordre nouveau repérable dans l'architextualité proposée?

\section{La musique comme " objet de valeur » : dispositifs techniques et pratiques sociales}

\subsection{De la patrimonialisation des musiques de variétés}

Le passage d'un média à l'autre et les mutations des écritures médiatiques peuvent s'observer dans le cadre du mouvement de patrimonialisation de la culture populaire et de la variété musicale. Ce que Le Guern (2012) nomme "l'obsession pour les nouveaux patrimoines", comme les musiques populaires, s'explique selon lui principalement par trois facteurs : technologique, sociologique et anthropologique. Dans ce contexte, la musique de variétés acquiert le statut de donnée mémorable en tant qu'elle entre dans des processus de valorisation patrimoniale et qu'elle devient objet de savoirs, de mémoire, de remémoration, voire de commémoration. Les institutions culturelles, mais aussi les médias prennent part à ces processus de légitimation et participent à " l'amenuisement de l'indignité culturelle des moins bien dotés en capital culturel qui partagent quelques goûts avec une part de plus en plus importante de la population » (Granjon et Combes, 2007, p. 23). C'est ainsi, par exemple, que la télévision peut être 
considérée comme étant une instance de reconnaissance et de légitimation « pour tous ceux qui ne font pas partie des milieux cultivés et ne bénéficient pas des réseaux d'informations courts et spécialisés »(Granjon et Combes, 2007, p. 23).

Les variétés, objet de notre étude, constituent un genre musical spécifique. Comme l'a souligné Hennion (1981), audelà de la voix et de la technique vocale parfois secondaires, la place du corps, la présence corporelle de l'interprète et son image y sont fondamentales:

"Sur le plan visuel, la physionomie de l'artiste, son maintien, sa démarche, sa tenue vestimentaire ont au départ une fonction expressive analogue à celle de la voix. II faut qu'il intrigue, qu'il force l'attention, donne aux gens qui le rencontrent envie de le connaître sur une simple impression visuelle » (p. 46).

La personnalité du musicien-interprète, son style, son histoire personnelle et les modalités de rencontre particulière avec le public (concerts) participent de la construction d'un personnage. Ce « charisme initial est ensuite développé tout au long du travail de production de la chanson: promotion, passages TV, presse, clips, affiches, concerts, pochettes de disques, etc. " (Dalbavie, 2012, p. 26.) Ces éléments conditionnent nécessairement la relation avec le public. Des travaux d'observation ethnographique comparative de la mise en scène des tombes de chanteurs populaires comme Georges Brassens d'une part et Claude François et Dalida d'autre part ont permis de montrer que ces mises en scène proposent une relation horizontale de l'ordre de la familiarité (pour Brassens) et une relation verticale de starisation (pour Claude François et Dalida). Dans les deux cas, le rapport au corps du chanteur repose sur le paradoxe entre proximité et distance, et les " médiations intermédiaires" (actions de promotion) entre les chanteurs et leurs publics permettent d'intensifier le moment de la rencontre sur scène qui constitue une sorte de "pèlerinage au corps » (Dalbavie, 2012, p. 31).

En nous inspirant de ces travaux, nous souhaitons montrer que les lieux numériques de la station fonctionnent eux aussi comme des lieux de rencontre. À l'instar des musées ou des tombes qui peuvent constituer des lieux de pèlerinage à l'image ou au corps (Dalbavie, 2012), nous faisons l'hypothèse que ces scènes numériques offrent, de par les spécificités du support et des composants scripturaux interactifs, des espaces de pèlerinage singuliers qui contribuent à ce processus de patrimonialisation. 
Radio musicale, prolongements numériques et régimes d'interactivité. Le cas de Nostalgie

\subsection{Hétérogénéité des pratiques de consommation musicale à l'ère du numérique}

L'expérience musicale provoque une émotion singulière et relie le mélomane à un ensemble de pratiques. Elle est à rapporter à l'environnement de l'écoute et aux objets. Avec le numérique, «la réception de la musique est sujette à un tournant marqué par l'hétérogénéité des pratiques de consommation des individus ॥ (Nowak, 2013, p. 1). En effet, avec la multiplication des équipements, des supports et le développement du $\mathrm{P} 2 \mathrm{P}$ ou du streaming, de nouveaux régimes d'usages adviennent et «redessinent les cadres de la consommation musicale " (Granjon et Combes, 2007, p. 10). La "numérimorphose " ouvre la voie à de nouveaux types d'appropriation et de relation qui jouent en faveur du morceau et non plus de l'album. Elle traduit un rapport renouvelé à la musique, mais aussi une "complexification des formes concrètes d'amateurisme qui avaient déjà été initiées durant le passage à la discomorphose » (ibid., p. 79).

La mise à disposition, les facilités d'accès, l'hétérogénéité des contenus, les possibilités de personnalisation, d'échange, de partage et de prescription génèrent de nouvelles formes d'intermédiation et décomplexent l'amateur. Ces modes de médiation modifient les processus de socialisation culturelle et les pratiques de consommation des médias qui proposent des offres de plus en plus segmentées. La dématérialisation par la numérisation octroie un autre statut au disque vinyle et au disque compact, qui deviennent des "objets culturels", concrets et collectionnables (Granjon et Combes, 2007) détenteurs de paratextes précieux (livrets, jaquettes, flyers, boîtiers...). De plus, les lieux et les moments de l'écoute se multiplient et les médias occupent, à ce titre, des positions d'accompagnants du quotidien qui ne sont pas menacés par cette démultiplication.

\subsection{Les radios musicales et leurs fonctions}

Le « dispositif d'intéressement » (Glevarec 2014) des radios musicales revêt quatre dimensions : la fonction d'identification recouvre la relation d'appartenance et de reconnaissance; la fonction de programmation concerne le flux de contenus musicaux susceptible d'intéresser l'auditeur; la fonction de découverte pose la radio comme source de découvertes de nouveautés; enfin, la fonction de "présence au présent » valorise l'écoute en direct, hic et nunc. Or internet « a amplifié les possibilités (souvent gratuites) de mise en relation entre les individus et la musique » (Glevarec 2014, p. 5) ${ }^{4}$, notamment par

${ }^{4}$ En novembre 2015, 24,3 millions d'internautes de 15 ans et plus ont consulté au moins un site ou une application de radio ou de musique depuis un ordinateur, un 
le téléchargement, et les radios musicales subissent la concurrence de "nouveaux médiateurs" de la musique (Deezer, Spotify) sur ses différentes fonctions.

Dans ce contexte, les radios musicales sont appelées à réagir, à s'adapter à l'environnement numérique et à adopter différentes stratégies:

« Certaines considèrent que ce contexte n'a pas d'incidence majeure sur les fonctions premières de la radio. D'autres réagissent en internalisant, via la création de webradios spécialisées (c'est le cas du groupe NRJ), cette expansion du champ musical et des moyens d'entrer en contact avec la musique. Enfin, il y a ceux qui investissent dans la radio sur Internet en spécialisant les radios selon des formats musicaux pointus »(Glevarec 2014, p. 13).

On peut donc, à terme, s'interroger sur les modalités par lesquelles les radios musicales peuvent, par leurs prolongements numériques, concurrencer le succès des plateformes musicales et examiner l'hypothèse que les lieux numériques radiophoniques mettent en œuvre de nouveaux " moyens d'entrer en contact avec la musique » (ibid.), par exemple en proposant des fonctionnalités proches de ces plateformes ${ }^{5}$.

\section{3. "Nostalgie.fr »: écriture radionumérique, fabrique d'un âge d'or et régime d'interactivite}

Nous voudrions porter une attention particulière aux modalités d'épaississement numérique d'une radio musicale thématique à partir d'un exemple, celui de la station Nostalgie. L'étude du cas s'inscrit dans un questionnement plus large qui interroge le passage d'une "écriture radiophonique » à une "écriture radionumérique » c'est-à-dire à une écriture "à informations ajoutées ", interactive, délinéarisée et flexible, incluant le matériau sonore initial: quelle place le sonore occupe-t-il dans cette transformation? Quelles propositions d'interactivité sont formulées en direction de ces auditeurs devenus radionautes ? En quoi favorisent-elles la fidélisation et

mobile ou une tablette. Cela représente plus de un internaute sur deux $(54,2 \%)$. Dans l'ensemble, $63,7 \%$ des visiteurs de sites et d'applications de «Radio \& Musique » les ont consultés au moins une fois depuis un mobile et/ou une tablette, soit 15,5 millions de visiteurs uniques (MÉDIAMÉTRIE, 2015, $<$ http://www.geste.fr/activites/linternet-global-radio-et-musique-0>).

${ }^{5}$ Cette piste de recherche autour de la relation entre les prolongements des radios musicales et les plateformes dédiées ne sera pas développée dans le cadre de cette contribution. 
Radio musicale, prolongements numériques et régimes d'interactivité. Le cas de Nostalgie

l'attachement à la station ? Et, enfin, ces nouvelles écritures modifient-elles les fondements ontologiques du média?

\subsection{Préambule méthodologique : sémiopragmatique du rapport d'inclusion et de la variation de supports}

Notre analyse ne repose ni sur des enquêtes d'audiences ni sur des observations de pratiques (voir Glevarec et Pinet, 2009) réalisées auprès des usagers, mais sur un examen des dispositifs dans leur matérialité, considérés comme étant des vecteurs de propositions. En effet, loin d'adopter une position qui relèverait d'un déterminisme technique, nous considérons qu'une prise de distance au regard de la nature et de l'étendue des possibilités d'interactivité proposées au radionaute est nécessaire pour appréhender ce passage de la radio au numérique selon l'angle des écritures.

La sémiopragmatique s'intéresse « en priorité aux grandes modalités de la production de sens et d'affects (vs au sens produit) ainsi qu'à leurs conditions de mise en œuvre »(Odin, 2000 , p. 56). Notre approche s'inspire des travaux qui mettent au centre de leur questionnement l'inscription du sens dans la matérialité du texte (entendu au sens large) et prennent en compte les différents niveaux de pertinence du sens lorsque celui-ci passe d'un média " traditionnel » - ici la radio - à un ou plusieurs autres supports matériels (smartphones, ordinateurs, tablettes) dépendants d'un régime de connectivité nécessitant, en totalité ou à un moment ou un autre de son utilisation, le passage par une connexion internet.

Nous partons de l'hypothèse que l'extension numérique de la radio modifie la production du sens, notamment par le rapport d'inclusion entre le média source et l'espace virtuel qui l'accueille. Entre le média accueilli et le média accueillant se met en œuvre une remédiation qui se traduit par des jeux d'influences réciproques.

Un média « offre, entre autres éléments, un dispositif ou [une] surface d'inscription avec des contraintes techniques susceptibles de modeler l'énoncé dans sa surface, sa temporalité, sa qualité et sa complexité de présentation et, du coup, ses conditions de réception "(Pignier, 2006, p. 9). Le sens n'est ni exclusivement dans l'énoncé ni exclusivement dans le média, il est le résultat d'une rencontre et émerge de différents niveaux de pertinence. Le, ou plutôt les sens possibles émergent d'une coénonciation dans un contexte particulier avec des contraintes et des propositions propres à un média (supports formel, matériel et erghodique) et à un genre de pratique médiatique. La part du sensible, c'est-à-dire les modalités par lesquelles sont mobilisés la vue, l'ouïe, mais 


\section{Séverine EQUOY HUTIN}

aussi le toucher par la manipulation et, plus globalement, le corps, intègre cette question :

«Nous envisagerons donc les effets sémiotiques d'une production multimédia, à travers des exemples d'application, comme l'articulation entre: son support formel; la page Web et l'écran en tant que surface d'inscription; son support matériel; les supports multimédias, l'ordinateur comme objets sensibles; le genre de pratique médiatique auquel il est intégré; l'association plus ou moins médiagénique d'un genre et d'un média ; l'exploitation stratégique des ambiguïtés de l'énoncé, pour qu'associé à un genre et un média, il ouvre une forme de vie spécifique; une esthésie grâce à laquelle la relation entre énonciateur et co-énonciateur prend forme. ॥ (Pignier, 2006, p. 9.)

Cette posture suppose de saisir un ensemble de traces en postulant que la surface d'exposition est dépositaire d'opérations et a fait l'objet d'actes d'inscription réalisés par son concepteur et matérialisés pour un public. Le public, " communauté de faire " ou " ensemble d'individus réunis par la mise en œuvre d'un système de modes de production de sens » (Odin, 2000, p. 60), est partie intégrante de l'analyse : il est pensé et inscrit dans la matérialité des textes qui portent des propositions de modes de lecture. Les modes d'écoute traditionnels de la radio se trouvent altérés par cette rencontre avec les nouvelles technologies: des modes de lecture-écoute sont proposés à l'internaute sur le mode d'une interactivité inscrite dans son horizon d'attente.

Le choix d'un support n'est jamais forfuit. Les regards sémiotiques qui interrogent «la façon dont les supports configurent les expériences énonciatives, perceptives deviennent plus que jamais nécessaires pour saisir les enjeux sociétaux contemporains " (Pignier et Mitropoulou, 2014, p. 16). La multiplication des supports s'inscrit dans une tension entre trois logiques: logique de substitution, logique de complémentarité, logique de préservation. Ainsi, les interfaces numériques qui font circuler les textes répondent à trois fonctions :

« une fonction de support matériel (volume, poids, maniabilité, taille) propre à l'objet matériel et mis en scène comme métaphore dans les interfaces graphiques des logiciels, des sites ; une fonction de support formel, mode d'organisation dans l'espace de l'écran et de la pageécran qui permet d'informer les textes, de les structurer dans l'espace de perception; une fonction de support 
Radio musicale, prolongements numériques et régimes d'interactivité. Le cas de Nostalgie

erghodique couvrant les modes d'interaction gestuelle, les fonctionnalités » (Mitropoulou et Pignier, 2014, p. 19).

Dans le passage d'un support matériel à un autre (du téléphone à l'ordinateur), d'un support formel à un autre (d'un site à une application ou à un réseau social), les textes s'exposent à des parcours de travail différents et à des contraintes formelles. Notre objectif est précisément d'interroger l'opérativité des supports et la manière dont ils médiatisent une source - ici le son diffusé en hertzien - de façon à mettre au jour la nature des effets engendrés par ce phénomène d'inclusion du matériau initial. C'est sous l'angle de la relation à l'interactivité, c'est-à-dire sous l'angle des modes d'appropriation et de ses traces, que nous choisissons d'aborder l'étude de cas qui suit.

3.2. Deux lieux numériques, deux dispositifs interactifs, deux régimes d'interactivité

L'hypothèse est que les deux lieux numériques étudiés proposent des régimes d'interactivité différents et des interactions différenciées avec la musique et considère la matérialité et les propositions de sens et d'action portées par les supports comme partie intégrante des environnements d'écoute.

3.2.1. Nostalgie : l'« agedorisation » des chansons de variété

Nostalgie a émis pour la première fois en 1983 à Lyon. La station fait partie du groupe NRJ Global, au même titre, par exemple que, NRJ, Rire et Chansons ou Chérie FM. Cette radio musicale commerciale et populaire réunit chaque jour environ 3034000 auditeurs. Elle est la deuxième radio musicale de France chez les 35-59 ans. La grille de programmation se structure en une succession de tranches horaires (par exemple "Nostalgie : $16 \mathrm{H}-20 \mathrm{H}$ ») et met en lumière quelques temps forts de la journée, comme l'émission matinale qui est la seule à disposer d'un titre à part entière : "Les matins qui chantent ", ou la rubrique "le Vinyle de $19 \mathrm{~h}$ ». Radio Nostalgie est orientée vers la glorification des artistes et des succès musicaux sur une période qui s'étend des années 1970 à 1990. La valorisation patrimoniale des genres musicaux et des interprètes représentatifs de cette période dont elle vise à entretenir la mémoire la constitue en espace commémoratif.

Dans un travail antérieur (Equoy Hutin, 2017), nous nous sommes intéressée aux prolongements numériques de la station sous cet angle de la dimension commémorative et des modalités de mise en scène d'une forme d'idéalisation du passé en tension entre permanence et modernité. Ce travail visait à observer la fabrique médiatique d'un âge d'or de la musique de variétés à partir de l'analyse du site internet de 
Nostalgie. L'interactivité promise par les supports numériques constitue, selon nous, un des facteurs d'une présence au monde, notamment en tant qu'elle propose une expérience de la musique qui diffère de l'expérience d'écoute radiophonique traditionnelle. Nous entendons donc, ici, compléter partiellement cette étude.

3.2.2. L'application mobile: un régime interactif de consommation instantanée

Les applications pour appareils portatifs (smartphones, tablettes) proposent des "services mobiles multimédia ». Elles sont le plus souvent soumises à un régime de connectivité (connexion à internet) et se situent dans une indépendance relative, en ce sens que l'appareil est repérable par les réseaux d'un point de vue commercial notamment. Excepté le coût lié à l'abonnement mobile ou internet, l'application est gratuite. Elle est téléchargeable sur les appareils disposant de systèmes d'exploitation mobile (Androïd, Windows phone, iOS...). Les services mobiles "sont configurés pour pouvoir être utilisés en milieu urbain et dans des espaces publics " (Licoppe et Zouinar, 2009, p. 6). Ils sont par définition voués à être utilisés dans des contextes de mobilité, si bien que leurs usages

\begin{abstract}
« requièrent que les utilisateurs articulent de manière publiquement intelligible des actions relevant de systèmes d'activité différents: d'un côté tout ce qui a trait aux mobilités urbaines (marcher, attendre dans une station de métro, prendre les transports publics, etc.) et de l'autre des pratiques culturelles (écouter de la musique, regarder la télévision, filmer) et communicationnelles (se parler au téléphone mobile, avec ou sans l'image visiophonique) » (ibid.).
\end{abstract}

Un des enjeux de l'écriture de l'application mobile est donc d'intégrer ces paramètres de façon à proposer une utilisation adaptée.

Le lancement de l'application Nostalgie propose un accès direct à la musique. Cet accès peut être différé par une annonce publicitaire dont la consultation peut être interrompue par un appui sur le signe " croix cerclée " situé en haut à droite de l'écran. L'écran d'accueil est divisé de haut en bas en quatre scènes horizontales (Figure 1). 


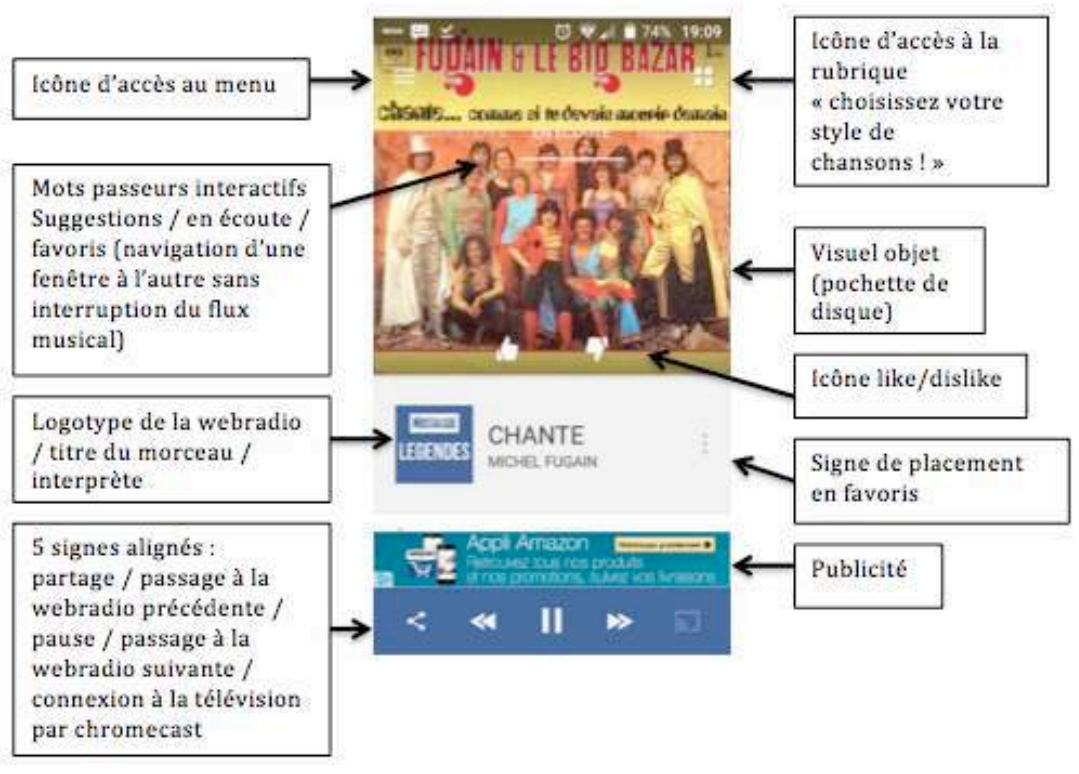

Figure 1. Capture d'écran de la page d'accueil après lancement de l'application Nostalgie

La première scène occupe la moitié de l'écran: elle permet de visualiser ce qui s'apparente à la pochette du 45 tours du morceau diffusé simultanément (Figure 1). On retrouve ici la logique de "morceau " (Granjon et Combes, 2007). Sur ce premier espace, quatre icônes permettent quatre premières formes d'interactivité par appui court, si l'on met de côté l'interactivité avec le téléphone lui-même, en cas par exemple de réception d'un SMS ou d'un appel entrant. L'usager a la possibilité :

1) d'accéder au menu ${ }^{6}$;

2) de choisir un style de chansons ${ }^{7}$ ("Choisissez votre style de Chansons!»; Figure 2) en sélectionnant une déclinaison thématique de la station parmi les 33 proposées $^{8}$ (Figure $3^{9}$ ) ;

3) d'écouter la programmation, de consulter les suggestions de la station ou ses favoris par les trois mots passeurs: " suggestions ", " en écoute ", "favoris ";

\footnotetext{
${ }_{7}^{6}$ Signe graphique constitué de trois traits horizontaux superposés.

${ }^{7}$ Signe graphique constitué de deux fois deux carrés blancs superposés.

${ }^{8}$ Exemples: Nostalgie Bob Marley, Nostalgie Le printemps qui chante, Nostalgie Chansons françaises, Nostalgie Hit parade 90, Nostalgie Saturday night, Nostalgie Fiesta, Nostalgie Funk, Nostalgie Best of 80's..

9 Le lecteur ne manquera pas de remarquer les doublons attribuables aux contraintes de capture du smartphone utilisé.
} 


\section{Séverine EQUOY HUTIN}

4) et de "liker » ou " disliker » (icône pouce levé/pouce baissé).

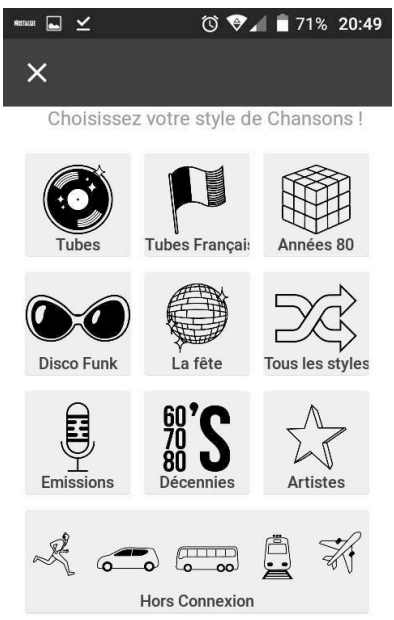

TOUTES LES RADIOS

Figure 2. Fonctionnalité « Choisissez votre style de Chansons !»

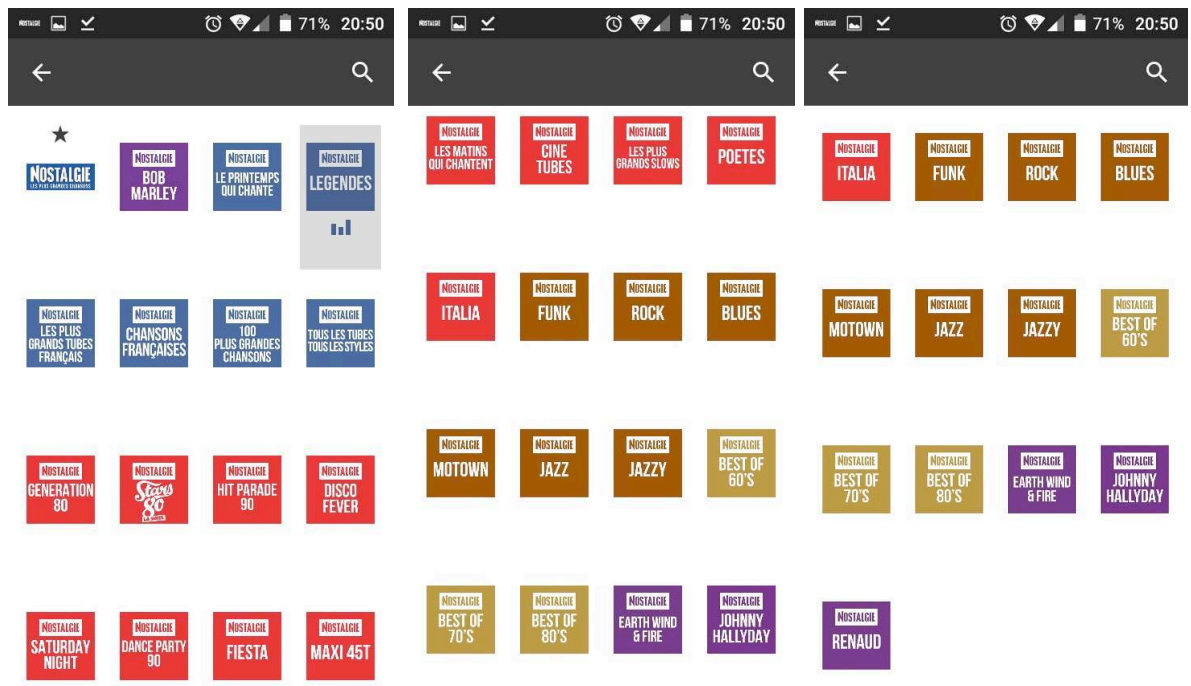

Figure 2. Captures d'écran montrant la diversité des propositions d'univers musical

Catégorisée selon un code couleur (brun pour les "styles " de musique, violet pour les "interprètes ", kaki pour les "best of "; les catégories bleues et rouges ne sont pas très clairement identifiables). Ici, la station en écoute, Nostalgie Légendes, est repérable grâce au fond gris et à trois bâtonnets verticaux dynamiques qui rappellent les égaliseurs dont peuvent être dotés les appareils (chaînes Hi-Fi) ou les logiciels d'écoute ou de traitement du son. 
Radio musicale, prolongements numériques et régimes d'interactivité. Le cas de Nostalgie

La deuxième scène reprend le logo miniaturisé de la station, mentionne le titre du morceau et le nom de l'interprète et présente une icône (trois points horizontaux) permettant de "mettre en favoris" le titre diffusé. Cette fonctionnalité est soumise à connexion par inscription. Ce pavé est déroulable par un mouvement tactile de bas en haut et laisse apparaître la programmation "à venir ». Les cinq prochains titres sont dévoilés selon une double logique : horizontale (par titre avec la miniature de la pochette du disque accompagnée du nom de l'interprète et du titre du morceau) et verticale (dans l'ordre de diffusion). Cette visibilité sur la programmation rappelle les interventions fréquentes des animateurs en direct qui, entre deux titres musicaux, annoncent la programmation à suivre, par exemple au cours de la prochaine demi-heure. Si le titre diffusé dans l'application ne correspond pas au goût de l'usager, celuici peut ainsi choisir de poursuivre l'écoute dans l'attente du prochain morceau ou de changer de webstation si la programmation à venir ne lui correspond pas. À la différence de l'écoute en hertzien où l'auditeur peut passer d'une station à l'autre, l'usager de l'application peut naviguer d'une webstation à l'autre selon une logique d'incitation à rester dans l'univers de la station mère.

Une troisième scène diffuse des annonces publicitaires. La disparition, par appui, de la publicité remet en visibilité la deuxième scène. La quatrième scène est organisée en une série de cinq signes graphiques alignés, qui permettent de partager via SMS/MMS, Bluetooth, email, Google Drive, Gmail (icône gauche " partage »), de naviguer d'une radio thématique à une autre selon une logique de défilement dans un ordre imposé, d'interrompre d'un simple appui l'écoute (pause) et, enfin, de se connecter, par exemple via Chromecast, à la télévision (rectangle connecté en bas à droite).

Le menu (Figure 4) est organisé en sept rubriques, déclinées sur fond bleu - conformément à la charte graphique de la station - avec en filigrane l'initiale de la station. 
Séverine EQUOY HUTIN

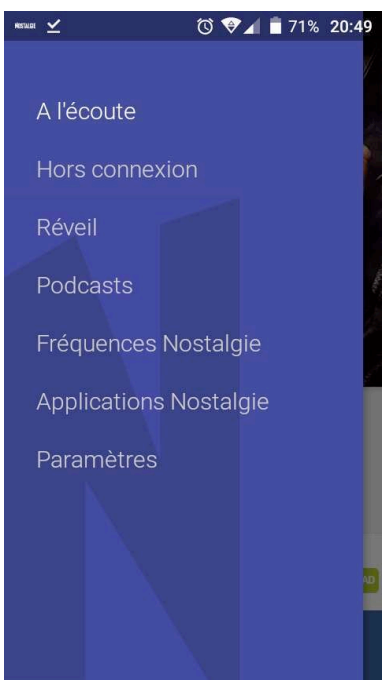

Figure 3. Menu de

l'application Nostalgie

Le Tableau 1 synthétise les fonctionnalités associées au menu. 
Radio musicale, prolongements numériques et régimes d'interactivité. Le cas de Nostalgie

Tableau 1. Rubriques déclinées dans le menu

\begin{tabular}{|c|c|}
\hline Entrées du menu & $\begin{array}{l}\text { Possibilités offertes via la manipulation } \\
\text { (appui, geste de défilement) }\end{array}$ \\
\hline $\begin{array}{l}\text { A l'écoute } \\
\text { (correspond à la } \\
\text { première page } \\
\text { affichée après } \\
\text { lancement de } \\
\text { l'application } \\
\text { (figure 1)) }\end{array}$ & $\begin{array}{l}\text { Choisir parmi } 33 \text { webstations prédéterminées } \\
\text { Identifier l'interprète et le morceau. } \\
\text { Interrompre l'écoute (mais le flux de diffusion continue) } \\
\text { Naviguer entre les webstations } \\
\text { Partager un titre } \\
\text { Se connecter à la télévision } \\
\text { Liker/disliker } \\
\text { Mettre en favoris } \\
\text { Consulter les stations favorites } \\
\text { Consulter les suggestions Nostalgie }\end{array}$ \\
\hline Hors connexion & $\begin{array}{l}\text { Consulter " mes contenus " et lancer une webstation } \\
\text { Télécharger une applications disponible (phonothèque) : } \\
\text { "Nostalgie génération } 80 \text { " qui peut migrer dans « mes contenus " }\end{array}$ \\
\hline Réveil & Se réveiller avec le jingle de Nostalgie \\
\hline Podcasts & $\begin{array}{l}4 \text { catégories: } \\
\text { - " Horoscope ": écouter son horoscope en choisissant dans un } \\
\text { empan des dix derniers jours } \\
\text { - "Star } 80 \text { " écouter des interviews de stars du film Stars } 80 \\
\text { (sponsorisé par la station), } \\
\text { - "Nostalgie »: écouter des extraits sonores (ici extrait d'interview } \\
\text { de Michel Polnareff) } \\
\text { - "Nostalgie ": écouter des extraits sonores de l'émission " Les } \\
\text { matins qui chantent " }\end{array}$ \\
\hline $\begin{array}{l}\text { Fréquences } \\
\text { Nostalgie }\end{array}$ & $\begin{array}{l}\text { Consulter pour connaître les fréquences hertziennes de la station } \\
\text { ville par ville (classement alphabétique) }\end{array}$ \\
\hline $\begin{array}{l}\text { Applications } \\
\text { Nostalgie }\end{array}$ & $\begin{array}{l}\text { Télécharger des applications des radios du même groupe (NRJ, } \\
\text { Chérie FM, Rire et chansons) }\end{array}$ \\
\hline Paramètres & $\begin{array}{l}\text { "Ecoute ": Partager l'écoute sur Facebook } \\
\text { "Utilisation »: Bénéficier de « bulles d'astuces (tips) pour } \\
\text { l'utilisation de l'application } \\
\text { " Nostalgie» : accéder au site internet, aux informations juridiques, } \\
\text { au numéro de la version de l'application }\end{array}$ \\
\hline
\end{tabular}

L'écriture de l'application repose sur une logique combinant facilité d'appropriation, rapidité d'utilisation, économie de manipulation et rationalité de la présentation: la mobilité du support formel répond donc à la mobilité de l'utilisation du support matériel, sans toutefois fonctionner exclusivement en vase clos. Le lien vers le site et l'accès à certaines fonctionnalités requièrent une mise en œuvre plus complexe (inscription, saisie d'identifiant et de mot de passe, identification via Facebook). L'interactivité est certes opérative, en faveur de la mobilité physique, mais elle vise également à mettre en avant la diversité des propositions d'augmentation musicales de la station, l'étendue des micro-univers musicaux proposés, qui peuvent pallier l'éventuelle insatisfaction de l'usager, et, enfin, les possibilités de personnalisation par la constitution de playlists. 
En outre, l'enjeu de fidélisation s'étend à l'ensemble du groupe NRJ, avec la possibilité de télécharger les applications d'autres stations du groupe. Les informations visuelles occupent l'écran et recouvrent essentiellement une fonction illustrative et esthétique. Même si un gain sur le plan des connaissances encyclopédiques par identification est présent (mention du nom de l'interprète, titre du morceau, reproduction miniaturisée de pochettes de 45 tours), il s'agit plutôt d'occuper l'écran. L'interactivité est conditionnée par l'affichage des titres à suivre, qui orientent la navigation. La continuité du flux, la fonctionnalité "réveil " et la consultation des fréquences Nostalgie rejoignent la fonction d'accompagnement du quotidien que recouvrent la radio en tant que média et le téléphone en tant qu'objet transportable. L'écoute des podcasts requiert, quant à elle, davantage de manipulation, et les tests effectués par nos soins ont permis de constater une difficulté d'accès, même après l'identification requise. Enfin, la fonction de partage permet par exemple à l'usager d'envoyer un SMS à un contact de son choix: "J'écoute CHANTE de MICHEL FUGAIN sur la radio NOSTALGIE LE PRINTEMPS QUI CHANTE. $\quad$ http://social.nostalgie.fr/nrj-webradio/v2-hits-91224801-22857 $»$.

Le lien hypertextuel qui clôt le SMS redirige instantanément vers la page "play.nostalgie.fr », qui propose à l'usager de se connecter pour partager et commenter avec ses amis la musique qu'il préfère.

Par rapport au matériau radiophonique, la station diffusée en hertzien constitue une proposition musicale parmi d'autres dans l'application. Elle n'est pas valorisée en tant que telle, car elle est mise sur le même plan que les 32 autres webradios. L'abondance de propositions musicales complète donc largement l'offre radiophonique source. La présence de la station se joue davantage sur le plan de la "station-marque " comme macro-univers musical de référence. Sur le plan de l'écoute, ce qui frappe, c'est la continuité du flux pendant les passages d'un écran-fenêtre à un autre, d'une entrée du menu à l'autre. Pendant le temps de navigation, seule une pression sur l'icône de pause ou un appel entrant peuvent venir perturber le flux musical. Ainsi, la musique succède toujours à la musique : ceci rappelle le concept même de radio musicale fondé sur l'ininterruption du flux.

L'application Nostalgie accorde donc une primauté à la musique: les propositions de manipulation et d'actualisation d'un " pouvoir faire » (Mitropoulou, 2012) visent à permettre à l'usager d'adapter sa consommation musicale à son humeur, à ses goûts, à son environnement spatial et temporel. Ces possibilités s'actualisent dans un univers facilement accessible, 
Radio musicale, prolongements numériques et régimes d'interactivité. Le cas de Nostalgie

souple mais balisé, qui limite les possibilités de dispersion de l'usager et joue en faveur d'une fidélisation à la station.

Le site internet: interactivité et construction d'un univers musical

Contrairement à l'application, l'entrée sur la page d'accueil du site via l'adresse www.nostalgie.fr (Figure 5) ne déclenche aucun son. L'écoute radiophonique est une possibilité donnée à l'usager. Elle est localisée en bas de page et visible en permanence, quelle que soit la page du site consultée.

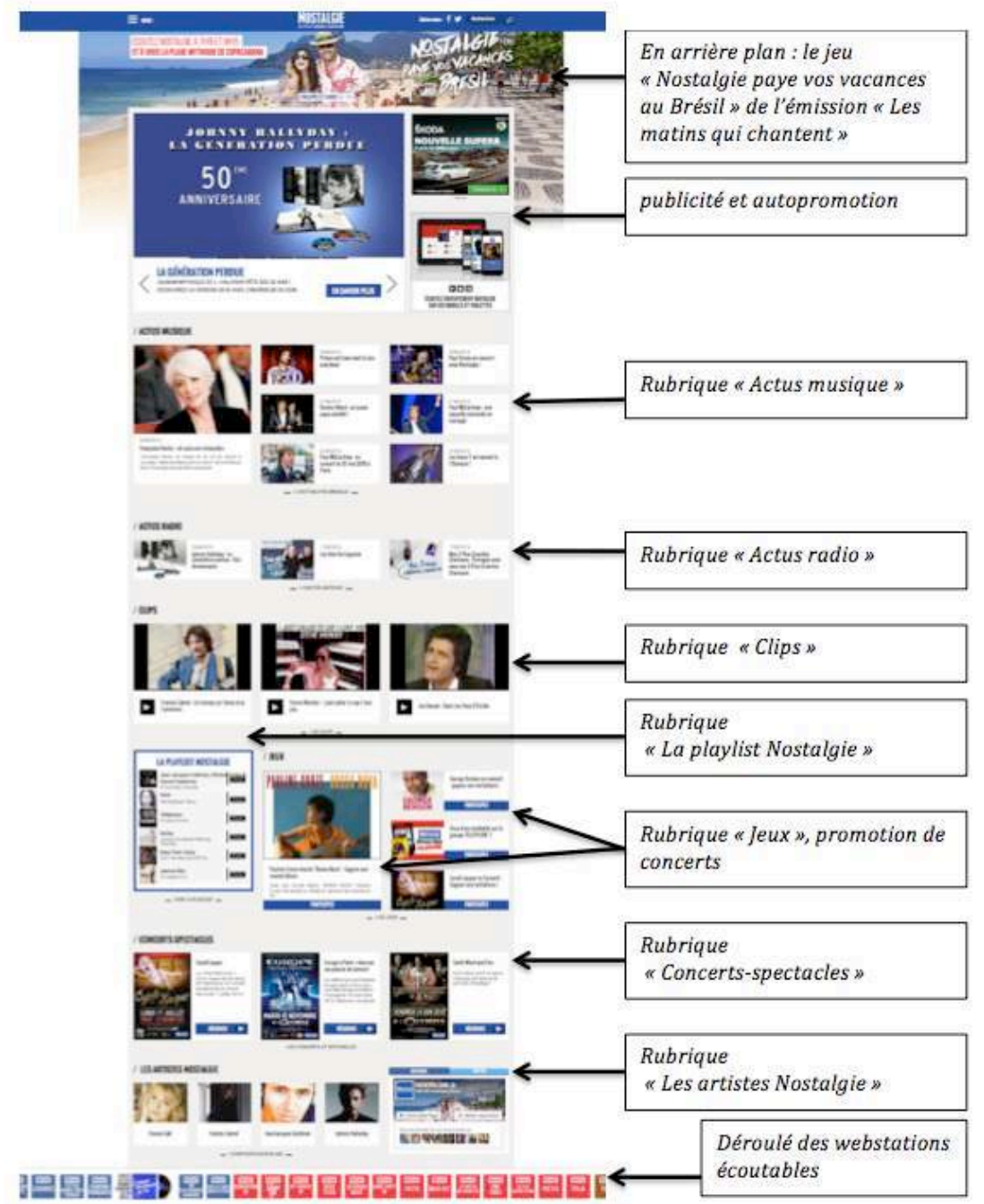

Figure 4. Capture d'écran du site www.nostalgie.fr. Page d'accueil commentée

La dimension promotionnelle est très présente sur la page d'accueil : publicité, promotion de l'émission vitrine et du jeu 
organisé par la station, promotion de la réédition d'un album, promotion de l'application, de la page Facebook et du compte Twitter de la station, promotion de concerts, etc. Ceci n'est pas étonnant quand on sait à quel point les sites internet sont soumis à des impératifs de fidélisation et de rentabilisation publicitaire (Rouquette, 2009).

Cinq rubriques se succèdent verticalement: "Actu Musique », "Actu radio ", " Jeux ", " Concerts-spectacles » et "Les artistes Nostalgie ». Ces rubriques sont présentées comme des vitrines et procèdent, selon les cas, d'une organisation en ligne ou en colonne. Pour chacune de ces rubriques, l'usager a la possibilité "d'en savoir plus » en cliquant sur le filet central (Figure 6).

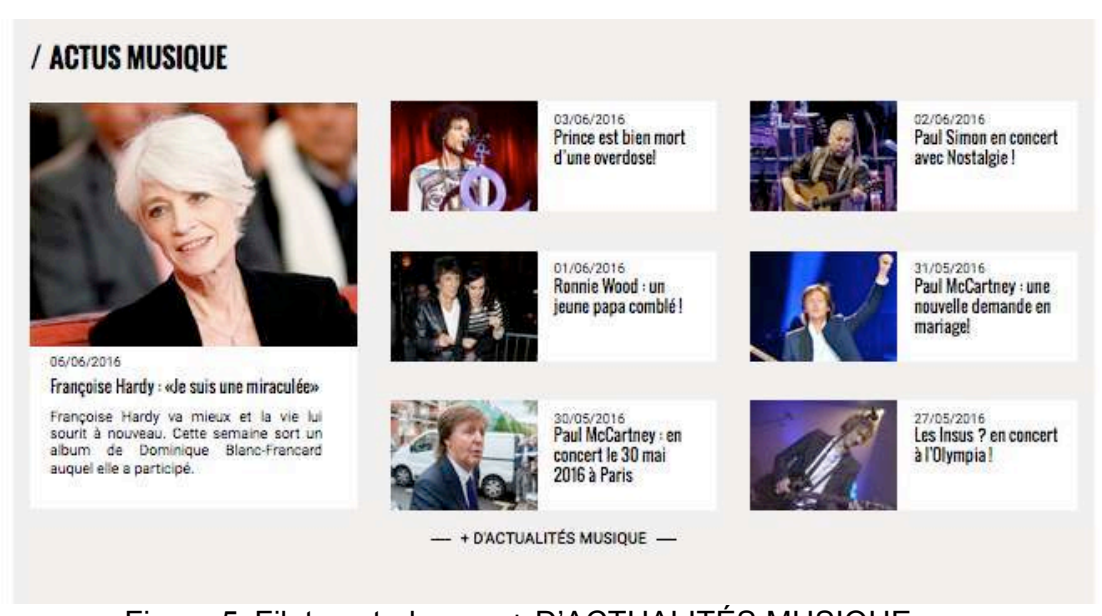

Figure 5. Filet central « — + D’ACTUALITÉS MUSIQUE —

À la différence des visuels proposés par l'application, le site internet octroie davantage de place aux corps en situation (concerts, soirées, émissions télévisées, déplacements divers...), dont on sait qu'ils sont fondamentaux dans la musique de variétés (Hennion, 1981). En outre, dans la rubrique "La playlist Nostalgie » (Figure 5), on retrouve les pochettes de disques ou de disques compacts qui rappellent l'application mobile.

La page d'accueil se présente comme une collection abondante de vignettes : elle accorde une place de choix aux artistes, aux photographies, aux extraits de vidéos d'époque ou récentes, aux jaquettes d'album ou de titre et aux affiches de concert qui fonctionnent ou qui sont appelés à fonctionner comme des "reliques" (Dalbavie, 2012) plus ou moins 
Radio musicale, prolongements numériques et régimes d'interactivité. Le cas de Nostalgie

anciennes. Elle évoque une page d'album photo, une collection de timbres ou une exposition muséale.

L'usager se voit proposer la possibilité de cliquer dans les fenêtres: les photographies comme les textes (titres et amorces) constituent des surfaces d'interactivité. Ainsi, en suivant le fil d'actualité de la chanteuse Françoise Hardy, qui occupe une place visuellement plus importante et préoriente donc la navigation, l'usager est dirigé vers une page mise en scène à la manière d'un article journalistique, contenant la même photographie et un article consacré majoritairement à la santé de la chanteuse (Figure 7). Au terme de cet article, l'usager découvre des liens vers les "dernières actus " de l'interprète, fonctionnant sur la même association photo/texte, puis trois liens promotionnels et un espace de commentariat. Sur la partie droite de l'écran, on peut voir une publicité puis un lien vers un espace dédié à la chanteuse, qui renvoie à la rubrique "Tous vos artistes" (menu) et propose une biographie, les articles " actu » qui lui sont consacrés, des vidéos, des photographies, l'inventaire des albums de l'interprète. Enfin, deux vignettes renvoient aux dernières « actus » de la station. 
Séverine EQUOY HUTIN

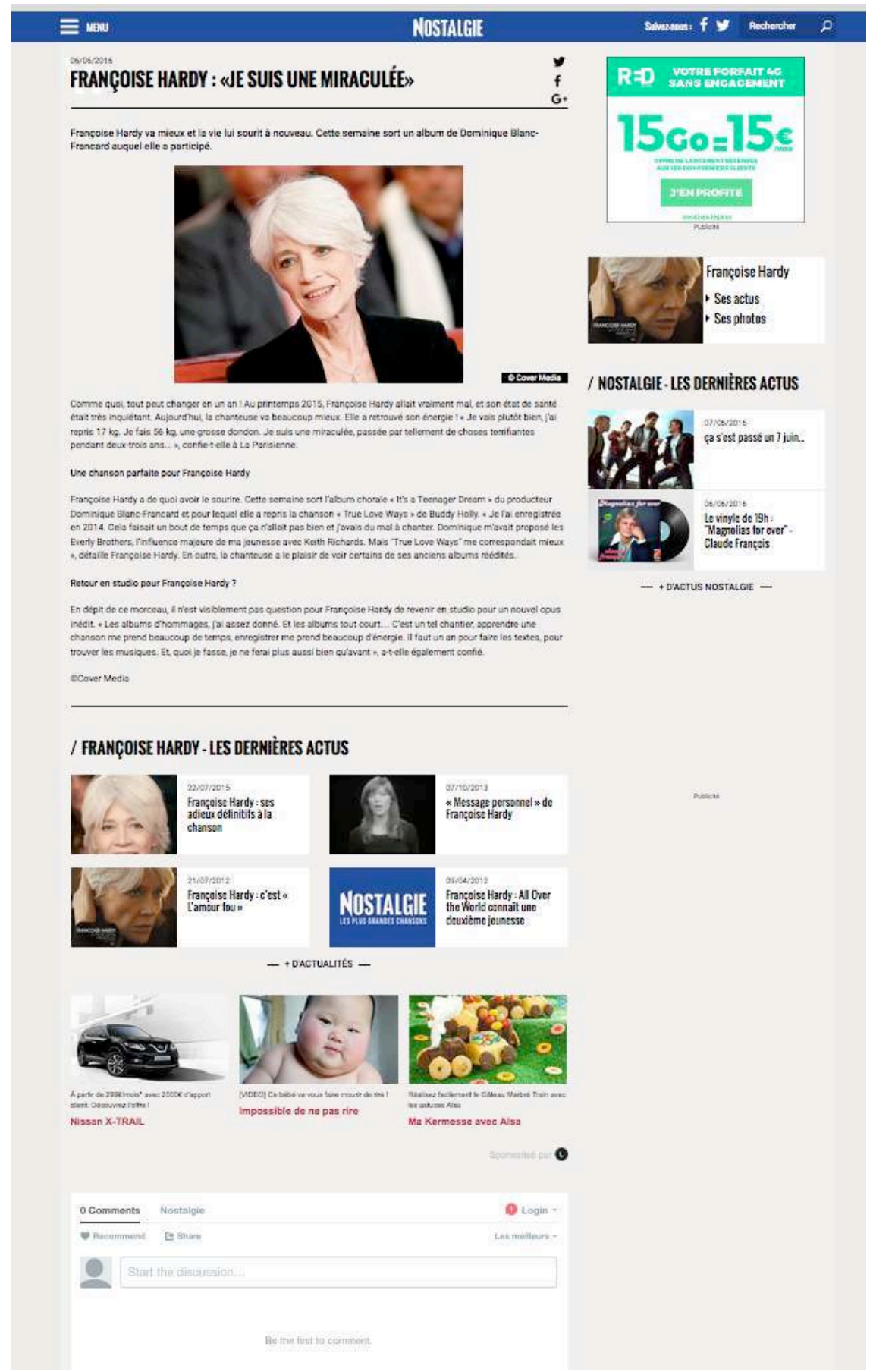

Figure 6. Page consacrée à l'actualité de Françoise Hardy

Le menu (Figure 8) est accessible par manipulation de l'icône située en haut à gauche de l'écran. Cette icône est identique à celle utilisée dans l'application. Ce menu se compose de six rubriques (en blanc sur le Tableau 2) 
Radio musicale, prolongements numériques et régimes d'interactivité. Le cas de Nostalgie

complétées par des signes passeurs vers une plateforme de chat, vers les applications téléchargeables disponibles et vers les réseaux socionumériques Twitter et Facebook. Chaque rubrique, excepté la rubrique "Jeux », comporte entre deux et huit « sous rubriques» (en gris sur les Figure 8 et Tableau 2). 
Séverine EQUOY HUTIN

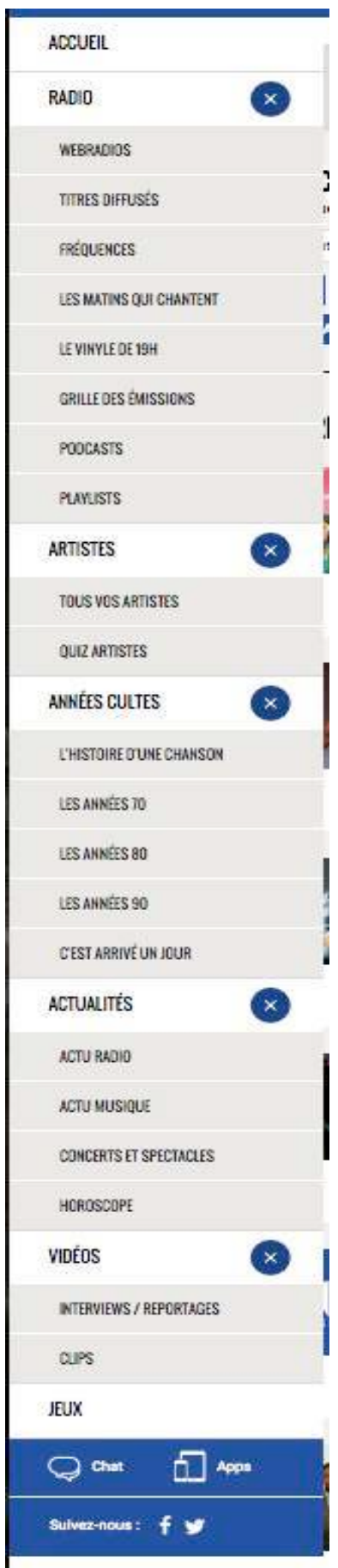

Figure 7. Menu du site déployé en rubriques et sous-rubriques 
Radio musicale, prolongements numériques et régimes d'interactivité. Le cas de Nostalgie

Tableau 2. Tableau des rubriques, menu du site internet www.nostalgie.fr

Rubriques/ Possibilités offertes via la manipulation

sous-rubriques

\begin{tabular}{|c|c|}
\hline \multirow{2}{*}{\multicolumn{2}{|c|}{$\begin{array}{l}\text { ACCUEIL } \\
\text { RADIO }\end{array}$}} \\
\hline & \\
\hline Webradios & Choisir parmi les 33 webradios \\
\hline Fréquences & $\begin{array}{l}\text { Connaître la fréquence hertzienne et de situer les fréquences } \\
\text { Nostalgie sur le territoire }\end{array}$ \\
\hline $\begin{array}{r}\text { Les matins qui } \\
\text { chantent }\end{array}$ & $\begin{array}{l}\text { Consulter la page de l'émission matinale, obtenir de l'« actu } \\
\text { antenne » }\end{array}$ \\
\hline Le vinyle de $19 \mathrm{H}$ & $\begin{array}{l}\text { Accéder à une collection organisée de vinyles (pochette avec } \\
\text { mouvement suggérant une exhumation, obtenir des } \\
\text { informations sur le titre du jour (jusqu'aux titres archivés } \\
\text { depuis le 09/10/2015), l'interprète et visualiser le clip via la } \\
\text { plateforme Dailymotion }\end{array}$ \\
\hline $\begin{array}{l}\text { Grille des } \\
\text { émissions }\end{array}$ & Consulter la grille de programmation \\
\hline Podcasts & $\begin{array}{l}\text { Écouter ou réécouter une émission ou une chronique } \\
\text { (horoscope) } \\
\text { S'abonner en installant iTunes }\end{array}$ \\
\hline Playlists & $\begin{array}{l}\text { Playlister un ou plusieurs titres parmi } 10 \text { titres mis à jour } \\
\text { quotidiennement } \\
\text { Écouter un extrait en ligne ou télécharger moyennant } \\
\text { paiement via iTunes }\end{array}$ \\
\hline \multicolumn{2}{|l|}{ ARTISTES } \\
\hline Tous vos artistes & $\begin{array}{l}\text { Rechercher un artiste et accéder à sa biographie } \\
\text { Commenter }\end{array}$ \\
\hline Quiz artistes & Jouer (chronoquizz) et tester sa connaissance d'un artiste \\
\hline \multicolumn{2}{|l|}{ ANNÉES CULTES } \\
\hline $\begin{array}{r}\text { L'histoire d'une } \\
\text { chanson }\end{array}$ & $\begin{array}{l}\text { Possibilité de connaître les conditions de création de la } \\
\text { chanson } \\
\text { Naviguer vers d'autres artistes via des liens hypertextes } \\
\text { Visualisation simultanée possible d'une prestation } \\
\text { audiovisuelle } \\
\text { Commenter }\end{array}$ \\
\hline Les années 70 & $\begin{array}{l}\text { Connaître les chansons, les artistes, les objets (le walkman), } \\
\text { les modes (le jean), les évènements médias (émissions } \\
\text { cultes) qui ont marqué la décennie }\end{array}$ \\
\hline Les années 80 & $\begin{array}{l}\text { Connaître les chansons, les artistes, les objets, les modes, } \\
\text { les évènements médias qui ont marqué la décennie }\end{array}$ \\
\hline Les années 90 & $\begin{array}{l}\text { Connaître les chansons, les artistes, les objets, les modes, } \\
\text { les évènements médias qui ont marqué la décennie }\end{array}$ \\
\hline C'est arrivé un jour & $\begin{array}{l}\text { Commémorer une date par sa mise en correspondance avec } \\
\text { un événement musical (sortie d'un album, censure ou } \\
\text { classement d'un titre) }\end{array}$ \\
\hline \multicolumn{2}{|l|}{ ACTUALITÉS } \\
\hline Actu radio & $\begin{array}{l}\text { Accéder à une sélection d'archives de la station (interviews } \\
\text { exclusives, photos de concerts privés) }\end{array}$ \\
\hline
\end{tabular}




\begin{tabular}{|r|l|}
\hline Actu Musique & $\begin{array}{l}\text { Accéder à des informations complémentaires sur l'actualité } \\
\text { des interprètes (article concernant un retour sur scène, la } \\
\text { préparation d'un album ou l'état de santé, une action } \\
\text { particulière comme la vente aux enchères d'objets } \\
\text { appartenant à un artiste) }\end{array}$ \\
\hline $\begin{array}{r}\text { Concerts et } \\
\text { spectacles }\end{array}$ & $\begin{array}{l}\text { Réserver des places de concert via le site } \\
\text { www.ticketmaster.fr } \\
\text { Connaître l'actualité de l'artiste concerné et les dernières } \\
\text { actus de la station. }\end{array}$ \\
\hline Horoscope & Connaître son horoscope de la journée \\
\hline Interviews/ & $\begin{array}{l}\text { Visionner des extraits d'interviews en studio ou d'émissions } \\
\text { en public animées par des chanteurs (8 pages) }\end{array}$ \\
\hline reportages & Visionner des clips (33 pages) \\
\hline Clips & Jouer pour gagner des places de concert \\
\hline JEUX &
\end{tabular}

La rubrique "Radio " se situe en première position et fait apparaître celle-ci comme un matériau source inclus dans l'offre et écoutable au même titre que les webradios dans la sous-rubrique éponyme. On retrouve les mêmes possibilités d'écoute et le même éventail de choix que dans l'application. Le choix d'une webradio fait accéder à plusieurs publicités audiovisuelles pendant le chargement annoncé de la webradio demandée. L'encart d'écoute (fond noir) rappelle alors les propositions de l'application avec la présence de la jaquette du titre, les possibilités de "liker/disliker », de " playlister » et les mentions (interprètes, intitulé). Les titres à venir dans la programmation sont également accessibles à partir des sept points alignés (rouge pour le titre en cours et blanc pour les titres suivants sur la Figure 9) et des flèches situées de part et d'autre de la jaquette. L'usager dispose d'un temps approximatif d'attente sur ces titres (" - de deux minutes ", " de cinq minutes », etc.). La partie gauche de l'encart laisse la possibilité de choisir entre les 33 webradios disponibles. 
Radio musicale, prolongements numériques et régimes d'interactivité. Le cas de Nostalgie

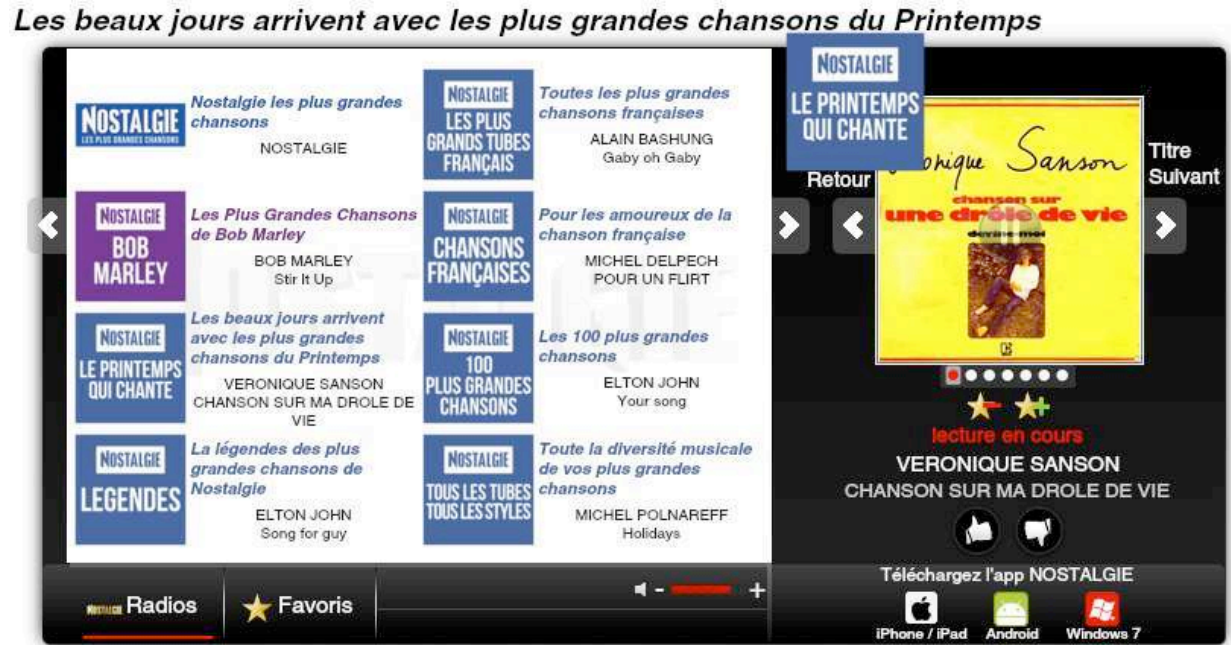

Figure 8. Capture d'écran pendant l'écoute de la webradio « Nostalgie Le printemps qui chante »

Si l'on retrouve certaines rubriques sur les deux supports (par exemple la rubrique "Fréquences »), celles-ci sont mises en scène différemment: dans la version "application », l'usager peut consulter les fréquences, alors que dans la version "site ", il peut visualiser et cliquer sur la carte de France pour découvrir la fréquence, le nom et le code postal de la ville choisie. La rubrique "Réveil » n'est, quant à elle, pas reprise dans la version "site », la fonctionnalité n'étant pas accessible hors connexion.

Le site propose donc un éventail de rubriques beaucoup plus large dans le menu. Ces rubriques recouvrent essentiellement une visée encyclopédique scénarisée sur un mode informatif (descriptif et/ou narratif) ou ludique : elles permettent de mieux connaître les artistes ("Tous vos artistes », "Quiz artistes »), les chansons et les années présentées comme cultes ( L'histoire d'une chanson », «Les années 70 », "Les années $80 »$, etc.).

Les autres rubriques du menu renvoient à d'autres actions : jouer, visionner des clips, des extraits d'émissions de variétés provenant des archives de l'INA (Institut national de l'audiovisuel). Le Guern (2012) souligne

« le rôle très actif qu'a joué le passage au numérique dans la reconfiguration des pratiques culturelles et mémorielles liées aux archives [...] le numérique favorise la transformation des contenus en archives, amplifie l'importance et la valeur des contenus vernaculaires, et modifie en profondeur notre rapport à la mémoire culturelle » (p. 28). 
Dans les rubriques "Actu musique » et "Clips ", les archives s'accumulent respectivement depuis 2001 (98 pages) et 2009 (33 pages). La rubrique "Les artistes Nostalgie » propose pour sa part des biographies de chanteurs plus ou moins connus et d'acteurs s'étant essayés ponctuellement à la chanson. Pour chaque bibliographie recensée, l'usager dispose d'un droit à commentaire sous réserve de s'identifier via Disqus, Facebook, Twitter ou Google).

Ce qui domine, c'est la possibilité d'accès à une abondance d'archives photo et vidéo (clips, interviews) qui font parfois doublon d'une rubrique à l'autre, voire à l'intérieur d'une même rubrique. Ces archives conditionnent l'interactivité: elles incitent l'usager à allonger son temps de consultation du site, par exemple en fragmentant sur un mode teasing les interviews en studio (Figure 10) et en proposant des liens soit vers la biographie de l'invité soit vers d'autres interviews d'un autre invité. La sous-détermination des titres choisis pour les vidéos (par exemple "Daniel Guichard sur Nostalgie (4/5) »), les différents cadrages et les différentes poses des captures choisies conduisent l'usager dans une dynamique de navigation. Cette dynamique repose également sur la rencontre avec un univers inaccessible en hertzien : les studios, les stars et les animateurs dans un contexte de travail. L'interactivité promet un accès sur le mode du faire voir ou du faire réécouter en faisant voir ce que l'usager ne peut habituellement pas voir ou ce qu'il a entendu, mais n'a pas pu voir. 
Radio musicale, prolongements numériques et régimes

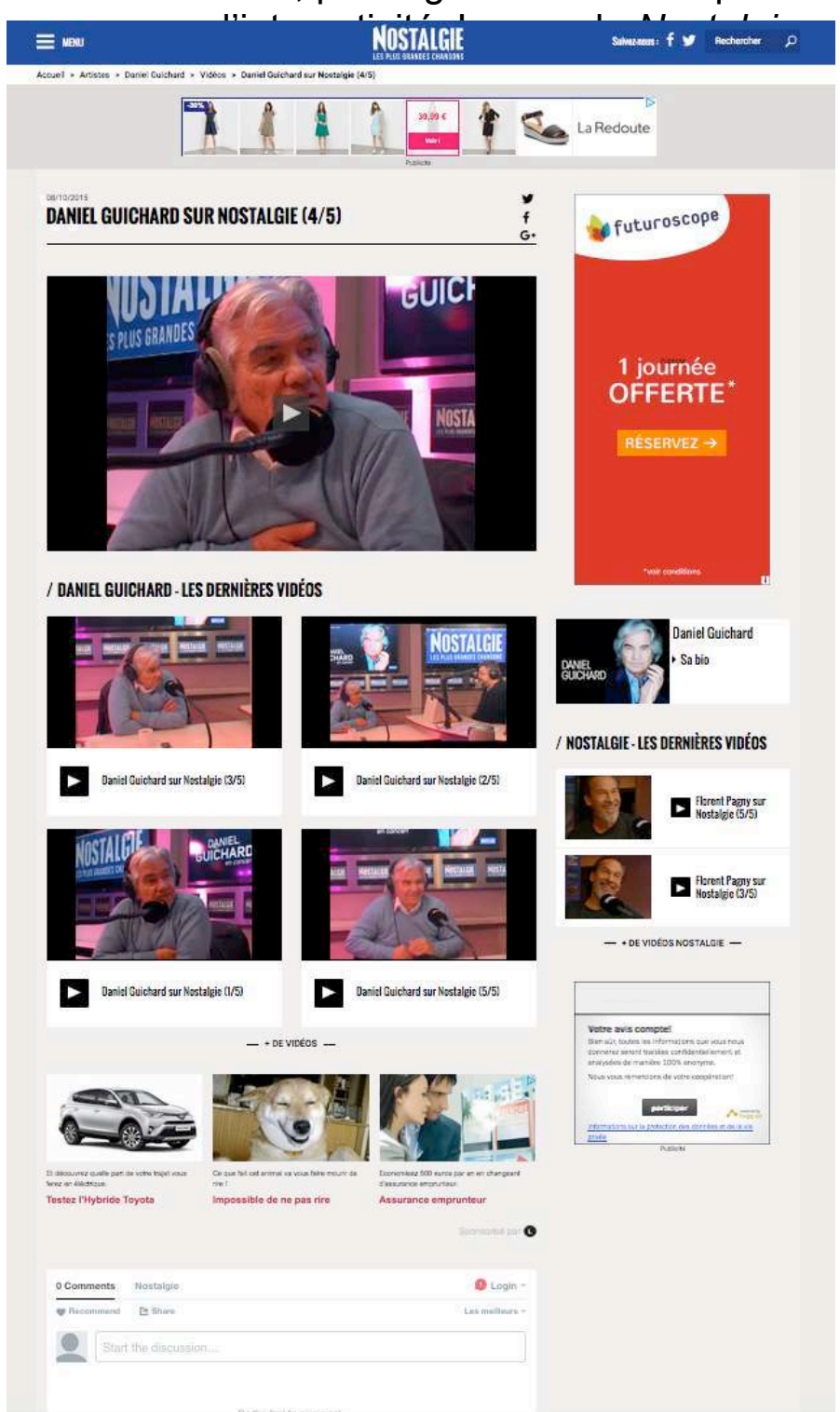

Figure 9. Interviews fragmentées en studio (rubrique « Vidéo, interviews/reportages)

Le site est enfin largement consacré à l'actualité et participe 
de la construction d'un âge d'or non pas révolu et regretté, mais pérenne et dynamique (Equoy Hutin, 2017): D'une part, l'actualité des stars (sortie d'une biographie, d'une réédition d'album, diffusion d'un portrait sur une chaîne télévisée, annonce d'un concert, informations privées allant au-delà du domaine musical) en s'attachant à articuler passé/présent/avenir dans le texte et dans l'image (Figure 8) ; d'autre part, l'actualité de la station qui s'autovalorise en tant que médiatrice. Cela dit, c'est davantage la station en tant qu'institution qui est valorisée, et non le média de diffusion. Au gré de sa déambulation, l'usager est ainsi mis en contact avec un univers artistique qui dépasse l'univers musical proposé par l'application.

\section{Conclusion}

Les deux lieux numériques de radio Nostalgie modifient l'expérience d'écoute proposée par la station sur les ondes. L'observation des marges de manœuvre interactive promises à l'usager permet de tirer quelques conclusions sur la place et le statut qu'y occupe la radio en tant que média et sur les représentations associées à l'objet de valeur «musique de variétés $"$.

Le processus de "ré-novation » de la radio offre d'autres vies éditoriales au matériau sonore initial: chaque support propose sa propre écriture en reconfigurant le flux initial et en s'inscrivant dans une généalogie des formes et des manifestations du sonore. L'outil porte « une mémoire du geste »(Souchier, 2004, p. 44), si bien qu'écouter la radio via un transistor, un téléphone ou depuis un site internet ne revient pas au même. Les processus combinatoires laissent leurs empreintes (Angé et Renaud, 2012) et modifient le statut du son: les deux supports formels étudiés incluent la diffusion de type hertzien et permettent une écoute en direct, soit de la " station mère ", soit des "webstations". Ces webstations dispensent un flux musical continu programmé, ce qui les rapproche de la diffusion hertzienne et de l'écoute " traditionnelle » de la radio. Toutefois, le degré supérieur de thématisation qu'elles proposent, tels des micro-univers sur lesquels on zoome, témoigne également de "niveaux de spécialisation » (Granjon et Combes, 2007, p. 3) et d'autres modalités de consommation liées à la réorganisation des contenus musicaux. Les webstations constituent une autre forme de direct, plus spécialisée sur un genre (Nostalgie Jazz), un artiste (Nostalgie Renaud), une époque (Nostalgie Best of 80 's) ou une situation (Nostalgie Fiesta). Ainsi, elles s'inscrivent dans une tendance qui vise à proposer un niveau supérieur de 
Radio musicale, prolongements numériques et régimes d'interactivité. Le cas de Nostalgie

spécification des goûts, à prolonger la durée d'écoute et à fidéliser. Ceci est d'autant plus remarquable que l'enquête sur les pratiques culturelles des Français réalisée en 2008 par le ministère de la Culture ${ }^{10}$ a permis d'une part de souligner la « montée de la culture de l'écran (Donnat, 2009) et d'autre part de montrer que la durée d'écoute de la radio en hertzien est globalement en baisse ${ }^{11} »$.

L'application mobile propose une ouverture qui reste proche du sonore et de la logique de flux. Les quelques contenus visuels proposés (jaquettes, logos) recouvrent une fonction certes informative, mais sont davantage destinés à occuper l'espace de l'écran et disparaissent lorsque l'appareil se met en veille. Le site internet positionne le son dans un ensemble encore plus étendu d'informations scénarisées sur des modes ludique (jeux) ou journalistique (articles, interviews filmées en studio). Le matériau source est ainsi relégué au rang de contenu facultatif (l'usager peut tout à fait consulter et naviguer sur le site sans jamais activer le son de son ordinateur). Si les parcours proposés dans l'application mènent toujours à du son, l'étendue du menu, la quantité d'informations et la nature des parcours possibles sur le site ne font pas de celui-ci une finalité.

Concernant les représentations associées à la « musique de variétés " dans le processus de patrimonialisation, nos observations permettent de distinguer deux régimes de consommation proposée par ces deux lieux numériques: un régime d'interactivité plus balisé à visée de consommation sonore personnalisée et instantanée pour l'application mobile et un régime d'interactivité à visée encyclopédique pour le site internet. Le régime d'interactivité de l'application appelle une consommation de flux plus dépendante d'une autre activité (prendre le bus, marcher dans la rue, patienter en salle d'attente). Par exemple, comme l'a montré Pecqueux (2009) dans le cadre d'observations ethnographiques de pratiques d'écoute musicale mobiles en contexte urbain, "ville et musique sont co-gérées par les auditeurs-baladeurs » (p. 19). L'expérience urbaine est conditionnée en partie par l'écoute musicale et réciproquement. La diversification des contenus et le degré de spécialisation introduit permettent une adaptation aux goûts de l'usager, aux spécificités de l'environnement

\footnotetext{
${ }^{10}$ Les résultats de l'enquête 2008 publiée dans l'ouvrage Pratiques culturelles des Français à l'ère numérique (La Découverte/Ministère de la Culture et de la Communication) sont disponibles sur le site en ligne: <www.pratiquesculturelles.culture.gouv.fr>, dernière consultation le 20 mars 2017.

${ }^{11}$ Entre 1997 et 2008, une baisse de deux à trois heures hebdomadaires selon les tranches considérées a en effet été constatée : 2 heures pour les 35-44 ans; 3,6 heures pour les $45-54$ ans ; 3,1 heures pour les 55-64 ans. Rappelons, comme indiqué plus haut, que Nostalgie est la deuxième radio musicale chez les 35-59 ans.
} 
sonore, de l'instant, de l'action et des affects qui lui sont associés. Le site internet appelle un cheminement autrement engagé en relation avec une autre forme d'immersion dans un univers artistique ciblé.

En déambulant physiquement ou virtuellement, l'usager construit un parcours dans un outil d'écriture écrit qui comprend " nécessairement une représentation de l'activité à laquelle il est dédié " (Souchier, 2012, p. 90). Ces deux régimes traduisent ainsi deux formes de vie et deux représentations de l'objet de valeur "musique »: ils modifient ou prolongent l'expérience du son par une expérience multisensorielle interactive qui mobilise l'ouïe, la vue et le toucher. Ces parcours dotent la musique de variétés d'une présence au monde différente. Nowak (2013) souligne que «les pratiques d'écoute sont toujours une question d'instant » (p. 40) et la musique en tant qu'objet esthétique ne peut être considérée en dehors de son environnement d'écoute. Les observations de Nowak ont montré que les supports d'écoute possèdent des qualités intrinsèques et permettent des accès à la musique différenciés. Ceci est d'autant plus remarquable à l'heure de l'éclectisme technologique et de la multiplication des contextes d'écoute. Les équipements nomades et l'ordinateur multitâche participent d'une " musicalisation de la vie quotidienne " (Donnat, 2009, p. 17). Les contenus musicaux se transportent et s'inscrivent dans des temps et des espaces singuliers. Si la finalité de l'application mobile reste le son, le site met à disposition un univers qui se déploie au-delà de la musique en tant qu'agencement sonore. L'abondance de reliques visuelles et audiovisuelles, les possibilités d'action (réservations de billets de spectacles, gain en participant à des quiz) et l'effet "puits sans fond " des actualités - qui n'en sont plus au fil du temps alimentent la dimension documentaire et culturelle du site. Or cette épaisseur n'est éprouvable que par un engagement et un temps consacré de la part de l'usager, un " voyage à travers des procédures écrites " (Souchier, 2012, p. 93), une dynamique de choix et une déambulation fondée sur des gestes d'activation-manipulation de textes mobilisant l'attention.

Les lieux numériques proposés par les objets interactifs peuvent enfin être envisagés comme des lieux d'exposition, de pèlerinage et des supports de publicisation plus ou moins pérennes qui participent du processus de patrimonialisation. Celui-ci implique « l'identification des traces, leur conservation et, enfin, leur exploitation, généralement par leur présentation, leur mise en visibilité selon différentes ressources » (Le Guern, 2012 , p. 8). II pose la question de la mémoire, de la remémoration en régime numérique: la musique de variétés devient un objet de mémoire dont les traces s'organisent et se 
Radio musicale, prolongements numériques et régimes d'interactivité. Le cas de Nostalgie

répartissent dans un environnement fragmenté et en renouvellement constant (réactualisations, mises à jour) qui s'affranchit du temps et de l'espace. Le processus de patrimonialisation s'opère ainsi à partir de cet amoncellement de données que la station, en tant qu'institution, prend en charge et dont elle se fait médiatrice. Comme l'a montré PatrinLeclère (2013), " les prolongements numériques des médias imprimés et audiovisuels [...] les conduisent à réfléchir au "capital" que constitue leur image, à penser leur potentiel de diversification à partir d'une politique dite de "produits dérivés" pour tenter d'assurer leur pérennité » (p. 18). Ainsi, faire du numérique et de l'interactif revient à construire un univers dédié qui bénéficie à la station en tant que marque. Les propositions de spécialisation s'apparentent à la fonction d' "experte » qu'acquiert la radio : "Pour certains, c'est la disponibilité même d'Internet qui pourrait être la cause d'un intérêt nouveau de la radio, non plus "découvreuse" mais "experte". La radio guiderait un auditeur perdu dans le champ culturel et informationnel " (Glevarec, 2014, p. 12). Les lieux numériques d'une radio comme Nostalgie peuvent à ce titre être considérés comme étant des sémiothèques complémentaires, à la fois repères et repaires pour l'auditeur attaché à la musique de variétés des années 1970 à 1990.

\section{RÉFÉRENCES BIBLIOGRAPHIQUES}

ANGÉ C. et RENAUD L., 2012, « Les écritures émergentes des objets communicationnels », Communication \& langages, $\mathrm{n}^{\circ} 174$, pp. 89-909.

BOLTER J.D. et R. GRUSIN, 2000, Remediation: Understanding new media, Cambridge, MIT Press.

BONACCORSI J., 2013, "Approches sémiologiques du web », dans C. BARATS (dir.), Manuel d'analyse du web, Paris, Armand Colin, pp. 125-146.

CHAUVIN VILENO A. et S. EQUOY HUTIN, 2016, "Radio augmentée, radio enrichie. De la transposition des émissions radiophoniques consacrées à l'Histoire sur le web: circulation, altération, transmission », RadioMorphoses, $\mathrm{n}^{\circ} 1$, $<$ http://www.radiomorphoses.fr/index.php/2016/05/02/radioaugmentee-radio-enrichie/>, dernière consultation le 19 mars 2017. 
CROZAT S., B. BACHIMONT, I. CAILLEAU, S. BOUCHARDON et L. GAILLARD, 2011, "Éléments pour une théorie opérationnelle de l'écriture numérique », Document numérique, vol. 14, pp. 9-33.

DALBAVIE J., 2012, "Entre patrimoine et mémoire collective, la tombe de Georges Brassens », Questions de communication, $\quad \mathrm{n}^{\circ} 22$, <http://questionsdecommunication.revues.org/6864>, dernière consultation le 20 juin 2016.

DELEU C., 2006, Les anonymes à la radio, Usages, fonctions et portée de leur parole, Bruxelles, De Boeck/INA.

DONNAT O., 2009, "Les pratiques culturelles des Français à l'ère numérique. Éléments de synthèse 1997-2008 », Culture études, $\mathrm{n}^{\circ} 5$, p. 1-12.

EQUOY HUTIN S., 2017, Nostalgie sur le web: construction d'un âge d'or de la variété française et internationale à l'ère de la postradio, Le temps des médias, $\mathrm{n}^{\circ} 27$, pp. 75-96.

GLEVAREC H, 2014, "Le propre de la radio. Fonctions radiophoniques et nouveau statut de la radio dans l'environnement numérique ", Le Temps des médias, $\mathrm{n}^{\circ} 22$, pp. 123-133.

GRANJON F. et C. COMBES, 2007, " La numérimorphose des pratiques de consommation musicale: Le cas de jeunes amateurs ॥, Réseaux, n 145-146, pp. 291-334.

HENNION A., 1981, Les professionnels du disque. Une sociologie des variétés, Paris, Métailié.

JEANNERET Y. et C. TARDY, 2007, L'écriture des médias informatisés. Espaces de pratiques, Paris, Lavoisier.

JEANNERET Y. et E. SOUCHIER, 2005, « L'énonciation éditoriale dans les écrits d'écran ", Communication \&langages, $n^{\circ} 145$, pp. 3-15.

LEJEALLE C., 2010, " Dans quelle mesure la TV sur mobile est-elle interactive?", tic\&société, vol. 4, $\mathrm{n}^{\circ} 1$, $<$ http://ticetsociete.revues.org/780>, dernière consultation le 31 mai 2016.

LE GUERN P., 2012, « Un spectre hante le rock... L'obsession patrimoniale, les musiques populaires et actuelles et les 
Radio musicale, prolongements numériques et régimes d'interactivité. Le cas de Nostalgie

enjeux de la "muséomomification" ", Questions de communication, $\quad \mathrm{n}^{\circ} 22$, <http://questionsdecommunication.revues.org/6820>, dernière consultation le 08 février 2016.

LICOPPE C. et M. ZOUINAR, "Présentation », Réseaux, vol. 4, $\mathrm{n}^{\circ} 156$, <www.cairn.info/revue-reseaux-2009-4-page9.htm>, dernière consultation le 28 mai 2016.

MANOVICH L., 2010, Le langage des nouveaux médias, Dijon, Les presses du réel.

MITROPOULOU E. et N. PIGNIER, 2014, «Introduction : interroger les supports? Matières, formes et corps ", Communication \& langages, $\mathrm{n}^{\circ} 182,2014, \mathrm{pp} .13-28$.

MITROPOULOU E., 2012, Écrans interactifs, promesses d'interaction, Interfaces numériques, vol. 1, $\mathrm{n}^{\circ} 1$, Paris, Lavoisier.

ODIN R., 2000, «La question du public. Approche sémiopragmatique », Réseaux, vol. 1, n 99, pp. 49-72.

PATRIN-LECLERE V., K. BERTHELOT-GUIET, V. JEANNEPERRIER, Y. JEANNERET et J.-L. MINEL, 2007, « Écrire le journal radiophonique: l'actualité naturalisée », dans Y. JEANNERET et C. TARDY, L'écriture des médias informatisés, Paris, Lavoisier, pp. 37-73.

PATRIN-LECLĖRE V., 2013, " Un média est-il une marque ? ", Communication, vol 32, <http://communication.revues.org/5027>, dernière consultation le $1^{\mathrm{er}}$ mai 2016.

PAQUIENSÉGUY F., 2011, "Multimédia et web 2.0: entre pratiques éditoriales et industries créatives », Les Enjeux de l'information et de la communication, vol. $12, \mathrm{n}^{\circ} 2$, $<$ www.cairn.info/revue-les-enjeux-de-l-information-et-de-lacommunication-2011-2-page-3.htmp.>, dernière consultation le 20 mai 2016.

PECQUEUX A., 2009, "Embarqués dans la ville et la musique. Les déplacements préoccupés des auditeursbaladeurs », Réseaux, vol. 4, n 156), pp. 49-80.

PIGNIER N., 2006, « Pour une approche sémio-pragmatique de la communication », Questions de communication, $\mathrm{n}^{\circ} 9$, 
<http://questionsdecommunication.revues.org/7945>, dernière consultation le 16 juin 2016.

RICHARD L., 1985, «De la radio et de l'écriture radiophonique ", Semen, $\quad \mathrm{n}^{\circ} 2$, <http://semen.revues.org/3733>, dernière consultation le 4 mars 2016

ROUQUETTE S., 2009, L'analyse des sites internet, Bruxelles, de Boeck.

SAINT MARTIN D. et S. CROZAT, 2007, "Écouter, approfondir... Perspectives d'usage d'une radio interactive», Distances et savoirs, vol. $5, \mathrm{n}^{\circ} 2$, <www.cairn.info/revuedistances-et-savoirs-2007-2-page-257.htm>, dernière consultation le 20 juin 2016.

SCHMITT B., 2009, "La radio au service de ses auditeurs », Groupe de recherches et d'études sur la radio, <http://www.grer.fr/upload/articles_en_ligne/La_radio_au_ser vice_de_ses_auditeurs.pdf $>$, dernière consultation le ${ }^{-} 19$ mars $20 \overline{17}$.

SOUCHIER E., 2012, " La "lettrure" à l'écran. Lire et écrire au regard des médias informatisés », Communication \& langages, $\mathrm{n}^{\circ} 174$, pp. 85-108.

SOUCHIER E., 2003, «Lorsque les écrits de réseaux cristallisent la mémoire des outils, des médias et des pratiques ",

$<$ http://www.interdisciplines.org/defispublicationweb/papers/1 8/1/1>, dernière consultation le 20 juin 2016. 